\title{
Aseguramiento de la calidad de doctorados en Educación en Chile
}

\author{
Quality assurance in education PhD in Chile \\ Ricardo Gaete Quezada
}

\begin{abstract}
RESUMEN
El objetivo del estudio es analizar las características de los programas de doctorado en Educación impartidos en universidades chilenas, desde la perspectiva de los procesos de aseguramiento de la calidad, considerando un enfoque cualitativo de investigación, mediante un análisis de contenido y un muestreo no probabilístico por conveniencia, para examinar los acuerdos de acreditación otorgados a cada programa por la Comisión Nacional de Acreditación. Los resultados obtenidos muestran que en la mayoría de los casos analizados estos programas no se encuentran acreditados o poseen un nivel de acreditación bajo, asociado con debilidades y oportunidades de mejora, las cuales se deben implementar a corto y mediano plazo. En conclusión, resulta imprescindible avanzar en la implementación de la reciente obligatoriedad de acreditar los doctorados en Chile, debido a la relevancia social y la necesidad de consolidar el ámbito educativo como un campo científico de excelencia en Chile.

Palabras clave: calidad de la educación, acreditación, educación superior, posgrados, grado de doctor.
\end{abstract}

\begin{abstract}
The objective of the study is to analyze the characteristics of the doctorate in Education programs taught by Chilean universities, from the perspective of quality assurance processes considering a qualitative research approach, through content analysis and a convenience non-probabilistic sampling, to examine the accreditation agreements granted by the National Accreditation Commission to each program. The results obtained show that in most of the cases analyzed these programs are not accredited or have a low level of accreditation, associated with weaknesses and opportunities for improvement, which must be implemented in the short and medium-term. In conclusion, it is essential to advance in the legal obligation to accredit all postgraduate programs in education, as is currently the case with undergraduate training, due to the social relevance and the need to consolidate the educational field as a scientific field of excellence in Chile.
\end{abstract}

Keywords: quality of education, accreditation, higher education, postgraduate, Doctor's degree. 


\section{INTRODUCCIÓN}

Según Rama (2006), la mercantilización de la educación superior en América Latina comenzó en la década de los sesenta, aumentando la oferta privada y disminuyendo la calidad, para favorecer una mayor cobertura a menor costo (Baeza, 2017; Muga, 2018; Rama, 2006). Asimismo, la formación docente se caracteriza por una fuerte orientación hacia la profesionalización, pero con bajos niveles de calidad, privilegiando el desempeño profesional en el sistema educacional formal y no formal (Gorostiaga, 2017; Rama, 2006). En las décadas recientes se ha registrado un fuerte aumento tanto de publicaciones como del número de investigadores dedicados al campo científico de la investigación educativa (Cabrera, 2013; Colina, 2011).

En lo relativo a los doctorados, Patiño (2019, p. 29) afirma que este tipo de programas de posgrado registra dos grandes tendencias a nivel mundial: a) armonización, asociada a la perspectiva más tradicional vinculada con la investigación disciplinar, y b) diversificación, relacionada con una nueva orientación hacia lo profesional en donde se promueva la complementariedad entre conocimiento científico y aplicado.

Según Corvalán, Falabella y Rojas (2011), Chile es ejemplo de un sistema universitario mercantil desregulado, que ha masificado su matrícula en la última década, siendo el primer país de América Latina en aplicar el pago de aranceles en las universidades estatales a comienzos de la década de los ochenta (Atria, 2012). Asimismo, los posgrados chilenos evidencian los impactos de las reformas mercantilistas, en virtud de las débiles regulaciones estatales y los escasos mecanismos de aseguramiento de la calidad de los programas de maestría y doctorado, especialmente en el área de educación, que en su mayoría no están acreditados (Baeza, 2017 y 2018; Walczak, Detmer, Zapata, Lange y Reyes, 2017).

Así, se analizan las principales características de los doctorados en Educación impartidos por universidades chilenas, desarrollando un análisis de contenido cualitativo de los Acuerdos de Acreditación otorgados por la Comisión Nacional de Acreditación (CNA) desde el año 2013 a la fecha, mediante un muestreo no probabilístico por conveniencia, para responder la pregunta de investigación: ¿Cuáles son las principales debilidades y retos que deben enfrentar los programas formadores de investigadores en educación en Chile desde la perspectiva del aseguramiento de la calidad?

Ricardo Gaete Quezada. Profesor del Departamento de Ciencias Sociales de la Facultad de Ciencias Sociales, Artes y Humanidades de la Universidad de Antofagasta, Chile. Es investigador de la temática de responsabilidad social universitaria desde 2008. Ha publicado más de 30 artículos científicos en revistas indexadas a nivel internacional, así como capítulos de libros en México, Colombia y Perú. Correo electrónico: ricardo.gaete@uantof.cl. ID: http://orcid.org/0000-0003-2359-2304. 


\section{REVISIÓN DE LA LITERATURA}

\section{Aseguramiento de la calidad en educación superior}

Según Salazar (2012, p. 29), el aseguramiento de la calidad en el ámbito universitario implica "un conjunto de mecanismos que apuntan al control, la garantía y promoción de la calidad", contemplando a la autoevaluación, la certificación pública otorgada por un ente externo y el diseño de planes de mejora como elementos esenciales.

En ese contexto, el aseguramiento de la calidad en las universidades es originario de los Estados Unidos (Rama, 2006), tanto la acreditación institucional como de programas de pre y posgrado, globalizándose mediante diferentes redes internacionales como la Red Internacional de Agencias de Acreditación (INQAAHE), creada en Hong Kong en el año 1991 (Atria, 2012; Salazar, 2012). Asimismo, la preocupación por la calidad de la educación superior en los países desarrollados data de los años noventa, extendiéndose rápidamente al resto del mundo (Adrogué, García, Carranza y Salto, 2019; Atria, 2012; Rama, 2006; Salazar, 2012).

Según Walczak et al. (2017), las fuertes presiones para mejorar su eficiencia, crecimiento y diferenciación, el aseguramiento de la calidad de las universidades, tiene entre sus finalidades el

Fomento y mejoramiento de la calidad; control de niveles mínimos de calidad; rendición de cuentas (accountability); información al público sobre estándares de calidad; certificación y acreditación; apoyo a la planificación institucional y del sistema; establecimiento de requisitos/direccionamiento del financiamiento; reconocimiento y rankings [p. 46].

Actualmente las universidades están inmersas en diferentes procesos de ajuste frente a los cambios sociales del siglo XXI (Lemaitre, 2018), en el cual el Estado asume nuevos roles para instalar agencias de aseguramiento de la calidad en América Latina (Rama, 2006). Según Miranda (2016, p. 53), "tanto en Chile como en muchos países del mundo se han iniciado procesos de regulación orientados a establecer mecanismos de aseguramiento de la calidad de la educación superior", situación que en Iberoamérica ha aumentado la producción científica de las universidades, comparada con las del resto del mundo (Muga, 2018).

Asimismo, el aseguramiento de la calidad contribuye al establecimiento de culturas de evaluación que garantizan el cumplimiento de estándares de desempeño (Atria, 2012; Silva, 2016), estableciendo ambientes de trabajo que las universidades deben aprender a gestionar más eficientemente (Lemaitre, Maturana, Zenteno y Alvarado, 2012; Miranda, 2016; Zapata, 2004). Lo anterior permitió instalar en la década de los noventa sistemas de aseguramiento de la calidad a nivel mundial (Atria, 2012; Lemaitre, 2018; Salazar, 2012).

En Chile la implementación del sistema de aseguramiento de la calidad de las universidades surgió durante los años noventa (Baeza, 2018; Walczak et al., 2017), relacionado inicialmente con la creación de la Comisión Nacional de Acreditación 
de Pregrado (CNAP) en el año 1999 (Poblete, Baeza y Droguett, 2018; Reich, 2003), transformándose posteriormente en la CNA en el año 2006 mediante la Ley 20.129 (Baeza, 2017; Lemaitre et al., 2012). También en el año 2006, a través de la Ley 20.129, se estableció el Sistema Nacional de Aseguramiento de la Calidad de la Educación Superior (SINACES), integrado por el Ministerio de Educación, el Consejo Nacional de Educación (CNED) y la propia CNA (Poblete et al., 2018).

Así, uno de los aspectos distintivos del caso chileno es la capacidad de autorregulación institucional mediante procesos tanto de acreditación de calidad institucional como de programas de pregrado y posgrado, desarrollando "políticas, mecanismos, procedimientos y acciones destinados a determinar si la institución está efectivamente avanzando hacia el logro de sus propósitos y objetivos" (Zapata, 2004, p. 146).

Según Walczak et al. (2017), el sistema de aseguramiento de la calidad chileno establece una evaluación de consistencia interna y externa respecto del conjunto de criterios de evaluación definidos por ley, en la cual las instituciones o programas sometidos a acreditación deben demostrar ante la CNA su capacidad de ajustarse a propósitos institucionales o a perfiles de egreso previamente definidos.

\section{Acreditación de posgrados en Chile}

Según Gorostiaga (2017), la investigación constituye el oficio de un académico cuyo propósito fundamental es la producción de conocimiento, considerando que

En el aprendizaje de ese oficio el doctorado aparece como la principal -y casi ineludible- instancia formal, se vuelve un medio de socialización en la cultura académica, en la cual juegan roles fundamentales actores como el director o tutor de tesis y el proceso de enseñanza y aprendizaje que se genera durante la elaboración de la propia tesis doctoral [p. 41].

Asimismo, Baeza (2017) afirma que los primeros programas de doctorado en Chile fueron creados entre 1968 y 1982 por universidades del Consejo de Rectores de Universidades Chilenas (CRUCH), fundamentalmente en el área de las Ciencias Naturales y Humanidades, con un bajo número de estudiantes matriculados. Luego, entre 1983 y 1989, se desarrolló la segunda etapa de la historia de los programas de doctorado en Chile, caracterizados por su escasa diferenciación y elitismo en el acceso.

Un hito importante fue la participación de la Comisión Nacional de Investigación Científica y Tecnológica (CONICYT), desarrollando programas de becas para estudiantes de doctorados acreditados en universidades del CRUCH e iniciando los procesos de acreditación de programas de posgrado en Chile en el año 1991 (Poblete et al., 2018; Reich, 2003).

En la última década aumentó significativamente la oferta de programas de doctorado y la cantidad de graduados (Tornero, Epstein y Vicuña, 2016; Baeza, 2017; Walczak et al., 2017; Muga, 2018; Poblete et al., 2018). No obstante, Walczak et al. (2017) señalan que si bien la formación de doctores en Chile ha aumentado diez 
veces en los últimos quince años, aún se encuentra por debajo del promedio de los países de la Organización para el Cooperación y el Desarrollo Económico (OCDE).

Tabla 1. Distribución matrícula programas de doctorado en Chile, por tipo de universidad.

\begin{tabular}{lccccccccccc}
\hline Tipo universidad/año & 2009 & 2010 & 2011 & 2012 & 2013 & 2014 & 2015 & 2016 & 2017 & 2018 \\
\hline CRUCH estatal & 1568 & 1756 & 1764 & 2028 & 2029 & 2163 & 2223 & 2372 & 2343 & 2455 \\
\hline CRUCH privada & 1560 & 1798 & 1951 & 1980 & 2074 & 2187 & 2342 & 2564 & 2554 & 2719 \\
\hline U. privadas & 610 & 501 & 337 & 463 & 50 & 575 & 607 & 609 & 643 & 756 \\
\hline Total & 3738 & 4055 & 4052 & 4471 & 4653 & 4925 & 5172 & 5545 & 5540 & 5930 \\
\hline
\end{tabular}

Fuente: Servicio de Información de la Educación Superior (SIES) (2019).

En la tabla 1 se observa un crecimiento sostenido en la última década de la matrícula de estudiantes en los doctorados chilenos en todas las áreas del conocimiento, mayoritariamente en universidades del CRUCH.

Otro hito importante ocurrió en el año 1999 con la creación de la Comisión Nacional de Acreditación de Posgrados (CONAP), continuando el trabajo iniciado por CONICY'T, consolidando una cultura de autorregulación de las universidades, alcanzando el 60\% de la oferta doctorados acreditados en todas las disciplinas científicas (Baeza, 2017; Poblete et al., 2018; Reich, 2003).

Según Poblete et al. (2018, p. 74), se ha consolidado una serie de buenas prácticas del Modelo de Acreditación de Doctorado de la CNA:

- Existencia de 18 comités de áreas o disciplinas científicas, encargados de desarrollar los procesos de acreditación de cada programa de doctorado.

- Comité consultivo de posgrado, especialmente para la definición y revisión de los criterios de evaluación respecto de los cuales se acredita cada programa.

- Existencia de recurso de reposición ante la CNA y de apelación ante el CNED que garantiza a los programas de doctorado un mayor nivel de objetividad de la evaluación.

- Existencia de formularios y guías de evaluación actualizadas, así como un apoyo permanente hacia los programas de doctorado de parte de la Secretaría Ejecutiva de la CNA y los comités de área a lo largo de todo el proceso de acreditación.

Actualmente, algunos desafíos para aumentar la calidad de los doctorados en Chile son: 1) mejorar las tasas de retención, deserción y graduación oportuna de sus estudiantes (Tornero et al., 2016); 2) aumentar la vinculación de los doctorados con el medio productivo (González y Jiménez, 2014; Walczak et al., 2017; Muga, 2018), y 3) asegurar la inserción laboral de los nuevos profesionales con grado de doctor (González y Jiménez, 2014). 
Sin embargo, Baeza (2017, p. 196) afirma que

...el 29\% de los programas de doctorado nunca se han sometido a procesos de acreditación en todas las áreas del conocimiento, o no han logrado acreditar de acuerdo con los criterios de evaluación de la CNA, establecidos en la Resolución Exenta DJ Nº 006-4 del año 2013.

El mismo Baeza (2017, p. 195) afirma que "la primera característica de la oferta de formación de doctorado en Chile es su heterogeneidad en términos de calidad, tomando la acreditación de la CNA como un indicador de calidad de la formación entregada".

Además, Poblete et al. (2018) afirman que la cobertura de acreditación de los doctorados en Chile promediaba 67\% durante el periodo 2011-2017, evidenciando un leve descenso en los últimos tres años de dicho periodo.

Según Corvalán et al. (2011), los doctorados en educación en Chile son un ejemplo de la ineficiencia del aseguramiento de la calidad a nivel de posgrado, evidenciando los efectos de la mercantilización en la educación terciaria chilena, especialmente en aquellas maestrías y doctorados que no están acreditados, afirmando que:

La educación superior chilena de posgrado, y en el caso particular del doctorado en educación, está regulada de manera laxa, lo que significa que no existe información suficiente que dé cuenta de la calidad de la oferta y que el principal elemento regulador del estado chileno -la acreditación- tiene un escaso efecto en orientar tanto la oferta institucional como a los demandantes de tales estudios [p. 18].

Según el Ministerio de Educación (2014), el único doctorado en Educación chileno que cumplía con los criterios de selección para participar en dicha investigación fue el doctorado en Ciencias de la Educación de la Pontificia Universidad Católica. Asimismo, Baeza (2017, p. 203) establece que

...hasta el año 2010 existían dieciocho programas de doctorados en el área de educación, de los cuales únicamente siete de ellos estaban acreditados por el tramo mínimo de tiempo (2-3 años), equivalentes al 3\% del total de doctorados en todas las áreas del conocimiento en el país.

Por ello, Gorostiaga (2017, p. 40) reconoce que la "tendencia general en los sistemas de educación superior latinoamericanos desde la década de 1990 ha sido el crecimiento de los programas de posgrado, incluyendo los del campo educativo, aunque no siempre bien integrados a la investigación”. Según Capocasale (2015, p. 41), la investigación en educación se puede definir como:

Una de las modalidades de Investigación Educativa cuyo objetivo es conocer para transformar o mejorar la práctica educativa. Su interés es docente e institucional. Su objeto es la práctica educativa ('el educar'). La realizan docentes-investigadores y otros actores sociales vinculados a la práctica educativa. Supone una mirada 'desde dentro', o sea que implica la aprehensión desde la experiencia. Su marco teórico general es dado por las Ciencias de la Educación en consistencia con el paradigma seleccionado desde la Investigación Educativa. 
La investigación en educación en Chile se caracteriza por una concentración en pocas universidades e investigadores, los que han contado con financiamiento estatal para el desarrollo de diferentes estudios (Palamidessi, Gorostiaga y Suasnábar, 2014). Sin embargo, Baeza (2018, pp. 218-219) afirma que los doctorados en Educación, junto con los programas del área de Ciencias Sociales, se ubican dentro de los "programas débiles" en el contexto de los doctorados chilenos, señalando que

La mayoría de estos programas no tiene experiencias en procesos de acreditación, y aquellos que se han sometido a una mirada externa por parte de CNA, han resultado no acreditados. A pesar de que el $75 \%$ de estos programas se implementan en universidades acreditadas, solo un $8 \%$ ha logrado un nivel mínimo de calidad certificado por CNA.

Políticas educacionales

Sistema educacional, gestión educacional a nivel local, resultados educacionales, políticas de educación superior.

Enseñanza y aprendizaje

Calidad y estrategias de instrucción y diseño de herramientas de enseñanza; desarrollo de habilidades y conocimientos matemáticos en el aula, incluidos en el currículo; Factores cognitivos, sociales y culturales en la enseñanza y el aprendizaje de conceptos básicos de matemáticas, ciencia y lenguaje; desarrollo y evaluación del lenguaje oral, de la escritura y la comprensión lectora; enseñanza de la lengua indígena mapudungun; Incorporación de modelamiento matemático y de STEM (ciencias, tecnología, ingeniería y matemáticas); Factores afectivos expuestos por estudiantes en clases STEM

Profesores y profesión docente

Formación inicial docente; trayectorias de los nuevos profesores; desarrollo profesional de los docentes; condiciones laborales de los docentes

Educación de la primera infancia

Formación inicial e identidad profesional de educadoras de párvulos; políticas públicas para educadoras de párvulos; transición y articulación entre la educación parvularia y básica; desarrollo de la agencia humana mediante prácticas pedagógicas; desarrollo profesional de educadoras de párvulos en servicio

Neurociencia, cognición y educación

Elementos cognitivos del aprendizaje matemático; Adquisición y aprendizaje del lenguaje; Aprendizaje perceptual y desarrollo de la experticia; bases neuronales del aprendizaje; Evaluación de funciones ejecutivas

Tecnología y educación

Condiciones de aprendizaje, motivación y prácticas pedagógicas en las aulas asociadas al uso de recursos TIC; Pensamiento pedagógico, dominios disciplinarios y didácticos de los docentes sobre las TIC; Uso de las TIC en el desarrollo de habilidades de alto orden, como son el pensamiento crítico y creativo, resolución de problemas, comunicación y trabajo en grupo; Manejo de las TIC en los contenidos curriculares y en el aprendizaje de niños y jóvenes en situación de discapacidad

Figura 1. Áreas y líneas de investigación en educación en Chile. Fuente: Centro de Investigación Avanzada en Educación (CIAE) (s. f). 
Según Corvalán y Rufinelli (2007, p. 23), entre los años 1995 y 2007 se había desarrollado un total de 1,311 estudios e investigaciones en educación de diversa índole (investigaciones, evaluaciones, artículos, entre otros), de los cuales el $24.2 \%$ de las iniciativas estudiaba la eficiencia/eficacia educativa, el 14.1\% era sobre pedagogía y el 13.9\% analizaba la formación y el perfeccionamiento docente. La figura 1 permite identificar seis áreas de investigación en educación en Chile, que orientan la formación de investigadores en educación en programas de posgrado.

Así, la investigación educativa debe relacionarse con una mayor responsabilidad social de las universidades como formadoras de investigadores en educación (MartíNoguera, Licandro y Gaete-Quezada, 2018), desarrollando líneas de investigación que posean una mayor pertinencia e impacto social de sus resultados (Miranda, 2016; Murillo y Perines, 2017).

\section{Metodología}

El objetivo del estudio es analizar las características de los doctorados en Educación impartidos por universidades chilenas, desde la perspectiva de los procesos de aseguramiento de la calidad, que cuenten a lo menos con un proceso de acreditación desarrollado por la CNA desde el año 2013 a la fecha.

La población de estudio está descrita en la tabla 2, compuesta por 19 universidades que ofrecen un total de 21 doctorados en el área de Educación, donde solo el $19 \%$ de ellos se encuentra acreditado por la CNA. Otra característica importante es que la mayoría de las universidades que oferta estos programas son instituciones de carácter privado $(79 \%)$.

La tabla 2 también identifica que los programas de doctorado más antiguos vigentes con alumnos datan del año 2004, pertenecientes a la Universidad Academia Humanismo Cristiano y a la Universidad de Playa Ancha de Ciencias de la Educación respectivamente, ninguno de los cuales se encuentra acreditado actualmente.

El estudio se realizó mediante un muestreo no probabilístico por conveniencia, incluyendo el examen de fortalezas y debilidades identificadas en los acuerdos de acreditación de los siete programas de doctorado en Educación en Chile identificados en la tabla 3, según la universidad que los imparte.

Los criterios de inclusión en la muestra del estudio son: 1) tener a lo menos un proceso de acreditación ante la CNA, y 2) considerar en el proceso de acreditación los lineamientos aprobados por la CNA mediante la Resolución Exenta DJ Nº 006-4 del año 2013.

Los acuerdos de acreditación fueron examinados mediante un análisis de contenido de tipo cualitativo, que implica "una técnica aplicable a la reelaboración y reducción de datos, que se beneficia del enfoque emergente propio de la investigación cualitativa (...) y del rigor de los criterios de regulación tradicionales" (Cáceres, 2003, 
Tabla 2. Oferta programas de doctorado en Educación en Chile.

\begin{tabular}{|c|c|c|c|c|c|}
\hline Universidad & Tipo & Programa de doctorado & Acreditación & Año inicio & Vigencia \\
\hline \multirow[t]{2}{*}{ Diego Portales } & \multirow[t]{2}{*}{ Privada } & En Educación Superior & No acreditado & 2010 & Vigente con alumnos \\
\hline & & En Educación & Acreditado & 2013 & Vigente con alumnos \\
\hline Bolivariana & Privada & $\begin{array}{l}\text { En Educación con mención en } \\
\text { aprendizaje transformacional }\end{array}$ & No acreditado & 2006 & Vigente sin alumnos \\
\hline $\begin{array}{l}\text { Academia de Humanismo } \\
\text { Cristiano }\end{array}$ & Privada & En Educación & No acreditado & 2004 & Vigente con alumnos \\
\hline La República & Privada & En Educación y Desarrollo & No acreditado & 2011 & Vigente sin alumnos \\
\hline SEK & Privada & En Educación & No acreditado & 2014 & Vigente con alumnos \\
\hline Andrés Bello & Privada & En Educación y Sociedad & No acreditado & 2018 & Vigente con alumnos \\
\hline $\begin{array}{l}\text { de Artes y Ciencias Sociales } \\
\text { (ARCIS) }\end{array}$ & Privada & $\begin{array}{l}\text { En Cultura y Educación } \\
\text { en América Latina }\end{array}$ & No acreditado & 2005 & Vigente sin alumnos \\
\hline Bernardo O'Higgins & Privada & En Educación & No acreditado & 2017 & Vigente con alumnos \\
\hline Alberto Hurtado & Privada & En Educación & Acreditado & 2013 & Vigente con alumnos \\
\hline del Biobío & CRUCH Estatal & En Educación & Acreditado & 2015 & Vigente con alumnos \\
\hline $\begin{array}{l}\text { Metropolitana de Ciencias } \\
\text { de la Educación }\end{array}$ & CRUCH Estatal & En Educación & No acreditado & 2005 & Vigente con alumnos \\
\hline $\begin{array}{l}\text { de Playa Ancha de Ciencias } \\
\text { de la Educación }\end{array}$ & CRUCH Estatal & Política y Gestión Educativa & No acreditado & 2004 & Vigente con alumnos \\
\hline De Los Lagos & CRUCH Estatal & En Educación Matemática & No acreditado & 2016 & Vigente con alumnos \\
\hline Católica de Chile & CRUCH Privada & En Educación & Acreditado & 2016 & Vigente con alumnos \\
\hline De Concepción & CRUCH Privada & En Educación & No acreditado & 2016 & Vigente sin alumnos \\
\hline Católica de Valparaíso & CRUCH Privada & En Didáctica de la Matemática & Acreditado & 2011 & Vigente con alumnos \\
\hline \multirow[t]{2}{*}{ Católica del Maule } & \multirow[t]{2}{*}{ CRUCH Privada } & En Educación & Acreditado & 2014 & Vigente con alumnos \\
\hline & & En Didáctica de la Matemática & No acreditado & 2018 & Vigente con alumnos \\
\hline $\begin{array}{l}\text { Católica de la Santísima } \\
\text { Concepción }\end{array}$ & CRUCH Privada & En Educación & Acreditado & 2014 & Vigente con alumnos \\
\hline Católica de Temuco & CRUCH Privada & En Educación & No acreditado & 2015 & Vigente con alumnos \\
\hline
\end{tabular}

Fuente: Elaboración propia, basado en Ministerio de Educación (2018).

p. 57), considerando como categorías y subcategorías de análisis a los criterios para la acreditación de programas de posgrado establecidos por la CNA (Baeza, 2017; Poblete et al., 2018), identificados en la figura 2.

Finalmente, los resultados obtenidos fueron triangulados mediante tres estrategias: 1) teoría expuesta en la revisión de la literatura; 2) datos cuantitativos de los acuerdos de acreditación, y 3) datos cuantitativos de fuentes secundarias, tales como páginas web de los doctorados o del Ministerio de Educación de Chile. 
Tabla 3. Doctorados de Educación en Chile sometidos a acreditación por la CNA.

\begin{tabular}{|c|c|c|}
\hline Universidad(es) & Programa & Acuerdo de acreditación \\
\hline Alberto Hurtado (UAH) y Diego Portales (UDP) & Doctorado en Educación & $\begin{array}{l}\text { Acreditado } 04 \text { años (2017-2021), Resolución } \\
\quad \text { N }^{\circ} 943 \text { del } 11 \text { de diciembre de } 2017\end{array}$ \\
\hline de Concepción (UDEC) & Doctorado en Educación & $\begin{array}{l}\text { No acreditado, Resolución Nº } 912 \\
\text { del } 20 \text { de junio de } 2017\end{array}$ \\
\hline $\begin{array}{l}\text { de Playa Ancha de Ciencias de la Educación } \\
\text { (UPLA) }\end{array}$ & $\begin{array}{l}\text { Doctorado en Política } \\
\text { y Gestión Educativa }\end{array}$ & $\begin{array}{l}\text { No acreditado, Resolución } \mathrm{N}^{\circ} 847 \\
\text { del } 21 \text { de octubre de } 2016\end{array}$ \\
\hline La Frontera (UFRO) & Doctorado en Educación & $\begin{array}{c}\text { No acreditado, Resolución N } 722 \\
\text { del } 15 \text { de octubre del } 2015\end{array}$ \\
\hline Pontifica Universidad Católica (PUC) & Doctorado en Educación & $\begin{array}{l}\text { Acreditado } 04 \text { años (2017-2021), Acuerdo de } \\
\text { Acreditación No } 904 \text { del } 8 \text { de mayo de } 2017\end{array}$ \\
\hline $\begin{array}{l}\text { Pontifica Universidad Católica de Valparaíso } \\
\text { (PUCV) }\end{array}$ & $\begin{array}{c}\text { Doctorado en Didáctica } \\
\text { de la Matemática }\end{array}$ & $\begin{array}{l}\text { Acreditado } 03 \text { años (2016-2019), Acuerdo de } \\
\text { Acreditación No } 815 \text { del } 7 \text { de junio de } 2016\end{array}$ \\
\hline $\begin{array}{l}\text { Católica del Maule (UCM), Católica Santísima } \\
\text { Concepción (UCSC), Católica de Temuco (UCT) } \\
\text { y del Biobío (UBB) }\end{array}$ & Doctorado en Educación & $\begin{array}{c}\text { Acreditado } 03 \text { años (2016-2019), Resolución } \\
\quad \text { NN }^{\circ} 861 \text { del } 18 \text { de noviembre de } 2016\end{array}$ \\
\hline
\end{tabular}

Fuente: Elaboración propia.

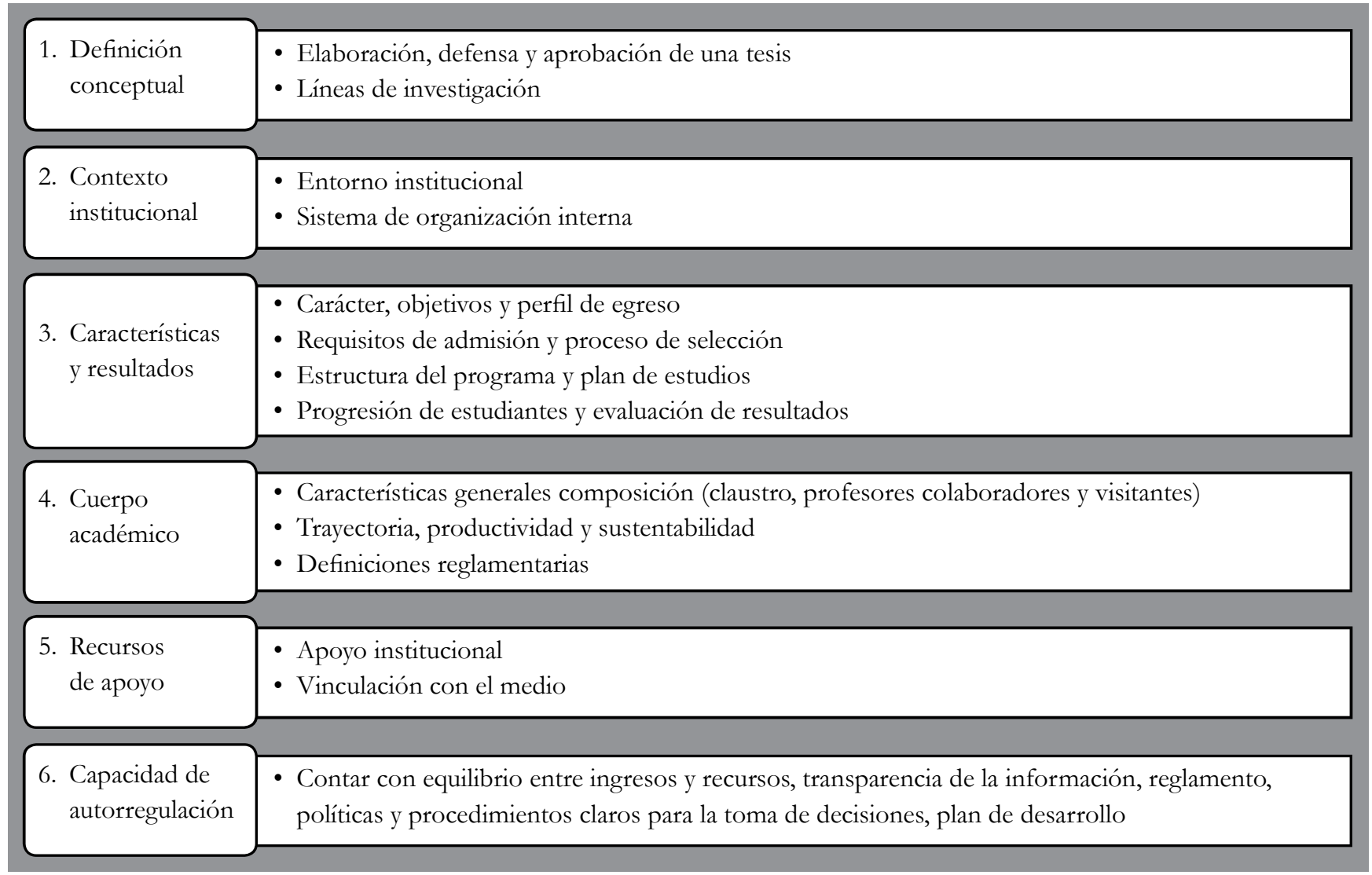

Figura 2. Criterios para la acreditación de programas de doctorado.

Fuente: Elaboración propia, basada en la resolución exenta de la CNA DJ Nº06-4 (2013). 


\section{Resultados}

\section{Definición conceptual}

En este primer criterio, cada doctorado debe explicitar sus líneas de investigación, transformándose en un aspecto esencial del aseguramiento de la calidad de los posgrados en Chile (Corvalán y Rufinelli, 2007; Murillo y Perines, 2017; Perines y Murillo, 2017; Adrogué et al., 2019). Los doctorados que cuentan con mayor cantidad de líneas de investigación identificadas en los acuerdos de acreditación son PUC y UFRO, con siete cada uno.

En la tabla 4 se expone una relación entre las líneas de investigación consignadas en los acuerdos de acreditación analizados y los lineamientos planteados por el CIAE identificados anteriormente en la figura 1.

Tabla 4. Líneas de investigación programas de doctorado analizados.

\begin{tabular}{lllllllll}
\hline & & & & & & UCM/UCSC/ \\
& UAH/UDP & UDEC & UPLA & UFRO & PUC & PUCV & UCT/UBB & Total \\
\hline Políticas educacionales & $\mathrm{X}$ & $\mathrm{X}$ & $\mathrm{X}$ & $\mathrm{X}$ & $\mathrm{X}$ & & & \\
\hline Enseñanza y aprendizaje & $\mathrm{X}$ & $\mathrm{X}$ & & $\mathrm{X}$ & $\mathrm{X}$ & & $\mathrm{X}$ & 5 \\
\hline Profesores y profesión docente & $\mathrm{X}$ & & $\mathrm{X}$ & $\mathrm{X}$ & $\mathrm{X}$ & $\mathrm{X}$ & 5 \\
\hline Neurociencia, cognición y educación & & & $\mathrm{X}$ & $\mathrm{X}$ & $\mathrm{X}$ & & 3 \\
\hline Tecnología y educación & & $\mathrm{X}$ & $\mathrm{X}$ & & & & 2 \\
\hline Educación de la primera infancia & & & & & & & & \\
\hline
\end{tabular}

Fuente: Elaboración propia.

Así, las líneas de investigación más destacadas en la tabla 4 son: políticas educacionales; profesores y profesión docente; enseñanza y aprendizaje. Por el contrario, sólo el doctorado de la PUC declara a la educación de la primera infancia como una línea de investigación en su oferta académica.

Adicionalmente, la educación intercultural es una línea de investigación en los doctorados de UFRO y la UCM/UCSC/UCT/UBB, ubicados en zonas del país con población perteneciente a pueblos originarios chilenos, evidenciando una importante pertinencia social de dichos posgrados (Miranda, 2016; Murillo y Perines, 2017).

\section{Contexto institucional}

En este criterio, cada programa de doctorado identifica fortalezas y debilidades del contexto institucional que alberga la formación de investigadores en educación en Chile, considerando dos sub-criterios: entorno institucional y estructura administrativa interna. 


\section{Entorno institucional}

Se analizan las políticas, normativas y estructuras institucionales de posgrado como soporte institucional para la formación de los futuros investigadores en educación. La mayoría de los programas incluidos cumplen con los diferentes aspectos considerados por la CNA, con excepción de los aspectos relacionados con la coordinación interna en dos doctorados:

No se observan mecanismos formales de coordinación con otros doctorados de la universidad ni con otras unidades académicas que alberguen programas relacionados a pedagogías [Doctorado PUCV, p. 3].

Si bien hay una coordinación administrativa entre las cuatro instituciones que imparten el doctorado, no se constatan mecanismos que aseguren la existencia de coordinación académica y de investigación entre los docentes [Doctorado UCM/UCSC/UCT/UBB, p. 3].

De acuerdo con la CNA, el 46\% de las universidades que imparten doctorados en educación no tiene acreditada institucionalmente el área de docencia de posgrado, como se observa en la tabla 5 . Asimismo, el $27 \%$ de las universidades no tiene acreditada el área de investigación, siendo ambas situaciones muy relevantes debido a que aseguran un entorno institucional de calidad para la formación de investigadores.

Tabla 5. Acreditación institucional universidades con doctorados en Educación, por áreas y años de acreditación.

\begin{tabular}{|c|c|c|c|c|c|c|}
\hline $\begin{array}{l}\text { Universidades / Áreas de } \\
\text { acreditación institucional }\end{array}$ & $\begin{array}{l}\text { Gestión } \\
\text { institucional }\end{array}$ & $\begin{array}{l}\text { Docencia } \\
\text { pregrado }\end{array}$ & Investigación & $\begin{array}{l}\text { Vinculación } \\
\text { con el medio }\end{array}$ & $\begin{array}{l}\text { Docencia } \\
\text { posgrado }\end{array}$ & $\begin{array}{l}\text { Años acreditación } \\
\text { institucional }\end{array}$ \\
\hline 1. Pontificia Universidad Católica & $\checkmark$ & $\checkmark$ & $\checkmark$ & $\checkmark$ & $\checkmark$ & 7 años $(2015-2021)$ \\
\hline 2. Universidad de Concepción & $\checkmark$ & $\checkmark$ & $\checkmark$ & $\checkmark$ & $\checkmark$ & 7 años (2016-2023) \\
\hline 3. Pont. U. Católica de Valparaíso & $\checkmark$ & $\checkmark$ & $\checkmark$ & $\checkmark$ & $\checkmark$ & 6 años $(2015-2021)$ \\
\hline 4. Universidad de la Frontera & $\checkmark$ & $\checkmark$ & $\checkmark$ & $\checkmark$ & $\checkmark$ & 6 años $(2018-2024)$ \\
\hline 5. Universidad Alberto Hurtado & $\checkmark$ & $\checkmark$ & $\checkmark$ & $\checkmark$ & $\checkmark$ & 5 años (2014-2019) \\
\hline 6. Universidad Diego Portales & $\checkmark$ & $\checkmark$ & $\checkmark$ & $\checkmark$ & $\checkmark$ & 5 años (2018-2023) \\
\hline 7. Universidad del Biobío & $\checkmark$ & $\checkmark$ & $\checkmark$ & $\checkmark$ & $\mathrm{X}$ & 5 años (2014-2019) \\
\hline $\begin{array}{l}\text { 8. Universidad de Playa Ancha de } \\
\text { Ciencias de la Educación }\end{array}$ & $\checkmark$ & $\checkmark$ & $\mathrm{X}$ & $\checkmark$ & $\mathrm{X}$ & 5 años (2016-2021) \\
\hline 9. Universidad Católica del Maule & $\checkmark$ & $\checkmark$ & $\mathrm{X}$ & $\checkmark$ & $\mathrm{X}$ & 5 años $(2015-2020)$ \\
\hline 10. Univ. Católica de Temuco & $\checkmark$ & $\checkmark$ & $\checkmark$ & $\checkmark$ & $\mathrm{X}$ & 4 años (2015-2019) \\
\hline $\begin{array}{l}\text { 11. Universidad Católica de la } \\
\text { Santísima Concepción }\end{array}$ & $\checkmark$ & $\checkmark$ & $\mathrm{X}$ & $\checkmark$ & $\mathrm{X}$ & 4 años $(2016-2020)$ \\
\hline
\end{tabular}

Fuente: Elaboración propia, basada en el buscador de acreditaciones de la CNA.

Del análisis de la tabla 5 se desprende que tres de los programas de doctorado en Educación en Chile (UAH/UDP, PUC, PUCV) pertenecen a universidades privadas, las que tienen acreditadas todas sus áreas institucionales por cinco años o más. La 
Universidad de la Frontera es la única institución estatal formadora de doctores en Educación que tiene todas las áreas de acreditación institucional aprobadas por la CNA.

\section{Sistema de organización interna}

En este sub-criterio se incluyen aspectos que utiliza cada doctorado tales como: sistema de dirección y gestión de los programas de doctorado, el perfil de los directivos de cada programa, los mecanismos de participación para los académicos que integran los claustros, y los mecanismos de comunicación y sistemas de información, transformándose en un ámbito que se encuentra bien desarrollado por la mayoría de los programas analizados en el presente estudio.

Excepcionalmente, en el acuerdo de acreditación del doctorado de la UFRO se identifica como una debilidad de su sistema de organización interna a la "alta concentración de actividades académicas y administrativas en la Directora del Programa” (p. 3), como un aspecto que ofrece una oportunidad de mejora en su funcionamiento.

\section{Características y resultados del programa de doctorado}

En este criterio se identifican aspectos tales como el perfil de egreso, admisión y selección de los estudiantes, programas y planes de estudios, progresión de los estudiantes y evaluación de los resultados académicos, concentrando la mayor cantidad de debilidades en los acuerdos de acreditación revisados durante la investigación.

\section{Objetivos y perfil de egreso}

En algunos acuerdos de acreditación se observan inconsistencias en la formulación de los objetivos de un programa de formación de investigadores a nivel avanzado:

Los seis objetivos específicos declarados, que deberían explicitar competencias claves del objetivo general, presentan una definición genérica, y se vinculan a propósitos de tipo institucional y no del programa de doctorado [Doctorado UDEC, p. 5].

Los objetivos planteados se refieren más a lo profesional que a lo académico, en su definición se incluye explícitamente la formación de profesionales. Asimismo, no se integra adecuadamente el énfasis en lo curricular y en lo latinoamericano "[...] el perfil de egreso alude a competencias como resultado del Programa y no como habilidades de los graduandos" [Doctorado UFRO, p. 3].

Así, la Resolución Exenta DJ Nº06-4 señala que cada programa debe contar con objetivos y perfil de egreso declarados explícitamente, debiendo ser consistentes entre sí, expresando con claridad el carácter, enfoque y orientación de cada doctorado. Según Núñez-Valdés y González (2019), es fundamental que los doctorados en Educación en Chile posean un perfil de egreso homogéneo, "considerando lineamientos internacionales, nacionales y las expectativas de los estudiantes de doctorado" (p. 163), como un estándar relevante de aseguramiento de calidad. 


\section{Admisión y selección de postulantes}

Un aspecto deficitario es la ausencia de mecanismos que permitan garantizar una mayor objetividad para evaluar a los postulantes a cada programa:

Se constatan algunos componentes discrecionales, en la forma en cómo se mide la experiencia académica y profesional del currículum y, también, cómo se mide la experiencia del recomendador, lo que incide directamente en el puntaje final del postulante [Doctorado UAH/UDP, p. 4].

El proceso de selección [...] cuenta con una pauta de evaluación que pondera cada uno de los criterios, sin embargo, ésta presenta ponderaciones discriminatorias al otorgar, por ejemplo, mayor puntaje aquellos estudiantes que provienen de universidades acreditadas por más de siete años [Doctorado PUC, p. 4].

En la tabla 6 se resumen los principales requisitos de ingreso o postulación exigidos a las personas que postulan a los doctorados en Educación considerados en nuestro estudio.

Tabla 6. Requisitos de ingreso/postulación en doctorados analizados.

\begin{tabular}{|c|c|c|c|c|c|c|c|}
\hline & UAH/UDP & UPLA & UFRO & PUC & PUCV & $\begin{array}{c}\mathrm{UCM} / \mathrm{UCSC} / \\
\mathrm{UCT} / \mathrm{UBB}\end{array}$ & Total \\
\hline Grado licenciado o magíster & $\mathrm{X}$ & $\mathrm{X}$ & $\mathrm{X}$ & $\mathrm{X}$ & $\mathrm{X}$ & $\mathrm{X}$ & 6 \\
\hline Dos cartas de recomendación & $\mathrm{X}$ & $\mathrm{X}$ & $\mathrm{X}$ & $\mathrm{X}$ & $\mathrm{X}$ & $\mathrm{X}$ & 6 \\
\hline Proyecto de investigación/carta de postulación & $\mathrm{X}$ & $\mathrm{X}$ & $\mathrm{X}$ & $\mathrm{X}$ & $\mathrm{X}$ & $\mathrm{X}$ & 6 \\
\hline Dominio idioma inglés (segunda lengua) & $\mathrm{X}$ & $\mathrm{X}$ & $\mathrm{X}$ & $\mathrm{X}$ & & $\mathrm{X}$ & 5 \\
\hline Aprobar entrevista personal & $\mathrm{X}$ & $\mathrm{X}$ & & $\mathrm{X}$ & $\mathrm{X}$ & $\mathrm{X}$ & 5 \\
\hline Certificar patrocinio o autorización laboral & $\mathrm{X}$ & $\mathrm{X}$ & $\mathrm{X}$ & $\mathrm{X}$ & & & 4 \\
\hline Notas y/o ranking licenciatura o magíster & $\mathrm{X}$ & $\mathrm{X}$ & & $\mathrm{X}$ & & & 3 \\
\hline Experiencia en investigación, trabajo escrito & $\mathrm{X}$ & & $\mathrm{X}$ & & & & 2 \\
\hline Rendir examen de admisión & & & $\mathrm{X}$ & & $\mathrm{X}$ & & 2 \\
\hline Certificado salud compatible con estudios & & & $\mathrm{X}$ & & & & 1 \\
\hline Acreditar financiamiento de los estudios & & & $\mathrm{X}$ & & & & 1 \\
\hline
\end{tabular}

Fuente: Elaboración propia, basada en las páginas web de cada programa.

La tabla 6 se elabora a partir de una revisión de las páginas web de cada programa, exceptuando al doctorado en Educación de la Universidad de Concepción, el cual no tiene disponible dicha información actualmente. Los aspectos más solicitados por los programas de doctorado son: grado de licenciado o magíster; presentación de dos cartas de recomendación de otros doctores, o investigadores, de reconocido prestigio, y carta del postulante exponiendo su proyecto de investigación a desarrollar durante el proceso de formación. Otros aspectos importantes son: acreditación del dominio del idioma inglés y aprobación de una entrevista personal. 
En la tabla 7 se exponen los principales resultados del proceso de selección que desarrolla cada doctorado incluido en nuestro estudio, desde la perspectiva del origen institucional y disciplinar de los postulantes aceptados.

Tabla 7. Resultados proceso de selección programas doctorados en Educación.

\begin{tabular}{|c|c|c|c|c|c|c|c|c|}
\hline & & UAH/UDP & UDEC & UPLA & UFRO & PUC & PUCV & $\begin{array}{c}\mathrm{UCM} / \mathrm{UCSC} / \\
\mathrm{UCT} / \mathrm{UBB}\end{array}$ \\
\hline \multicolumn{2}{|c|}{ Tasa de aceptación postulantes } & 12 & 29 & 28 & 53 & 25 & 45 & 49 \\
\hline \multirow{2}{*}{$\begin{array}{l}\text { Origen } \\
\text { institucional }\end{array}$} & Universidad nacional (\%) & 83 & 100 & 100 & 80 & 86 & 69 & 91 \\
\hline & Universidad extranjera (\%) & 17 & 0 & 0 & 20 & 14 & 31 & 9 \\
\hline \multirow{2}{*}{$\begin{array}{l}\text { Origen } \\
\text { disciplinar }\end{array}$} & Educación y afines $(\%)$ & 67 & 56 & 77 & 65 & 65 & 100 & 84 \\
\hline & Otras disciplinas $(\%)$ & 33 & 44 & 23 & 35 & 35 & 0 & 16 \\
\hline
\end{tabular}

Fuente: Elaboración propia, basada en los acuerdos de acreditación de cada doctorado.

Según la tabla 7, los doctorados en Educación chilenos muestran una baja internacionalización en cuanto al origen institucional de sus estudiantes, y solo el doctorado de la PUCV muestra un mayor porcentaje de alumnos extranjeros, debido a la especificidad del perfil de egreso que intenta formar en el ámbito de las matemáticas (Núñez-Valdés y González, 2019).

Sin embargo, algunos doctorados del estudio muestran una mayor apertura hacia un origen disciplinar más diverso, lo que establece una debilidad que plantea la necesidad de aplicar pruebas diagnósticas que evalúen competencias específicas relativas a cada programa:

Considerando la diversidad en la formación de origen de los estudiantes que postulan y se matriculan, el proceso de selección no contempla pruebas de conocimiento disciplinares e investigativos en el área. Asimismo, tampoco se considera evidenciar el manejo instrumental del idioma inglés, lo que no es consistente con las exigencias del plan de estudios, el cual contempla bibliografía obligatoria en dicho idioma [Doctorado UPLA, p. 4].

Se constata que se aceptan personas que no provienen del campo de la educación, pero no se advierte la existencia de cursos de nivelación [Doctorado UAH/UDP, p. 5].

Lo anterior queda confirmado en la tabla 6 , en la que se observa que únicamente la UFRO y la PUCV exigen rendir un examen de admisión a sus postulantes, destacando también que la demostración de experiencia previa en investigación solo es considerada por los doctorados de UAH/UDP y la UFRO, siendo necesario perfeccionar permanentemente los procedimientos aplicados para seleccionar a los futuros investigadores en educación (Corvalán et al., 2011; Miranda, 2016). 


\section{Estructura curricular}

La tabla 8 resume las principales características de los doctorados en Educación en Chile incluidos en nuestro estudio respecto del funcionamiento de su estructura curricular, según los acuerdos de acreditación de cada programa.

Tabla 8. Características estructura curricular doctorados analizados.

\begin{tabular}{lcccccccc}
\hline & & & & & & UCM/UCSC/ \\
& UAH/UDP & UDEC & UPLA & UFRO & PUC & PUCV & UCT/UBB \\
\hline Semestres de duración & 8 & 8 & 7 & 6 & 8 & 6 & 8 \\
\hline Núm. de horas directas e indirectas & 5280 & 6480 & 4860 & 3072 & 6600 & - & 6060 \\
\hline Tasa de graduación $(\%)$ & 25 & - & - & 64,7 & 44 & 53,8 & - \\
\hline Tasa de deserción $(\%)$ & 11 & - & - & 10,3 & 13,6 & - & 12,5 \\
\hline
\end{tabular}

Fuente: Elaboración propia, basada en acuerdos de acreditación de cada doctorado.

Según la información expuesta en la tabla 8, la duración de los programas de doctorado en Educación incluidos en nuestro estudio fluctúa entre los seis y los ocho semestres, con una cantidad de horas que supera las 4,000, con excepción del caso de la UFRO:

El programa declara una duración en horas presenciales y no presenciales de 3072 horas, lo que es inferior a las 3600 horas mínimas [...] que solicita la CNA en sus criterios de acreditación [Doctorado UFRO, p. 4].

Por otra parte, una debilidad de este sub-criterio observada en los acuerdos de acreditación es su inconsistencia con las líneas de investigación declaradas por cada programa:

Solo tres de las cuatro líneas de investigación declaradas presentan un correlato formativo dentro de la estructura curricular, a través de seminarios de carácter obligatorio, con excepción de la línea de educación y trabajo [Doctorado UAH/UDP, p. 5].

Respecto a los contenidos de los cursos obligatorios -que se circunscriben a las tres líneas formativas - no es claro cómo estos tributan a la obtención de las habilidades y competencias, ya que se evidencia ausencia de tópicos claves como: el análisis de dato y diseño (de la línea de política educacional) y evaluación (de la línea gestión educacional). Sobre la formación metodológica, los contenidos de los cursos que se circunscriben dentro de la línea de investigación educacional no incluyen una formación homogénea tanto en el ámbito cualitativo como cuantitativo [Doctorado UPLA, p. 5].

La oferta de los cursos de especialización no es consistente con las siete áreas temáticas que declara el programa, tampoco existen cursos de nivelación que permitan resguardar la diversidad disciplinar, que los estudiantes muestran al ingreso [Doctorado UDEC, p. 7]. 
También se observa como una debilidad importante en los acuerdos de acreditación a la inconsistencia entre lo declarado en los objetivos y el perfil de egreso, respecto de las asignaturas y cursos contemplados en la estructura curricular:

No se consignan actividades formales tendientes a habilitar a los estudiantes para la elaboración de proyectos de investigación, a pesar de ser un aspecto presente tanto en los objetivos como en el perfil de graduación [Doctorado PUCV, p. 5].

El perfil contempla la adquisición de competencias vinculadas a la creación de soluciones creativas a problemas educativos, a través de proyectos de investigación social y educacional, propósitos que no se contemplan dentro de la estructura curricular, ya que no se constatan cursos o actividades formativas que consideren la formación aplicada de los estudiantes [Doctorado UDEC, p. 6].

La estructura curricular no contempla adecuadamente el carácter interdisciplinario, latinoamericanizante y focalizado en currículum. No hay evidencia de suficientes actividades asociadas a estos aspectos del Programa [Doctorado UFRO, p. 4].

La mayor consistencia entre los objetivos y el perfil de egreso de cada doctorado, bien articulados con la estructura curricular y con las líneas de investigación desarrolladas por los profesores de cada doctorado, se transforman en las principales mejoras de corto y mediano plazo, en el contexto de sus mecanismos de aseguramiento de la calidad (Lemaitre et al., 2012; Miranda, 2016; Zapata, 2004).

\section{Cuerpo académico}

En este criterio se incluyen aspectos tales como: las características generales de los docentes, su trayectoria, productividad y sustentabilidad, así como la existencia de definiciones reglamentarias que regulen su quehacer.

La tabla 9 presenta el resumen de las características generales y la productividad del cuerpo académico de cada programa, a partir de la información proporcionada por la CNA.

Tabla 9. Composición y productividad del cuerpo académico, doctorados en Educación con acuerdo de acreditación.

\begin{tabular}{|c|c|c|c|c|c|c|c|c|}
\hline & & $\mathrm{UAH} / \mathrm{UDP}$ & UDEC & UPLA & UFRO & PUC & PUCV & $\begin{array}{c}\mathrm{UCM} / \mathrm{UCSC} / \\
\mathrm{UCT} / \mathrm{UBB}\end{array}$ \\
\hline \multirow{3}{*}{$\begin{array}{l}\text { Características } \\
\text { generales }\end{array}$} & Claustro de profesores & 17 & 12 & 14 & 21 & 33 & 9 & 19 \\
\hline & Profesores colaboradores & 17 & 10 & 3 & 5 & 22 & 2 & 16 \\
\hline & Profesores visitantes & 24 & 3 & 4 & 6 & 31 & 0 & 0 \\
\hline \multirow[t]{2}{*}{ Productividad } & Publicaciones Wos (ex ISI) & 2,5 & 3,4 & 0,44 & 1,57 & 1,9 & 0,76 & 0,77 \\
\hline & Proyectos Fondecyt & 0,9 & 1,3 & 0,21 & 0,95 & 1,06 & 1,11 & 1,15 \\
\hline
\end{tabular}

Fuente: Elaboración propia, basada en acuerdos de acreditación de cada programa.

Según la tabla 9, los doctorados en Educación analizados cumplen con el estándar mínimo de la CNA de siete académicos jornada completa adscritos a la propia 
universidad, mayoritariamente, en el claustro de profesores, quienes serán los encargados de las actividades docentes, dirección de tesis y administración del doctorado.

Una de las principales debilidades observadas en los programas de doctorado estudiados se relaciona con una mayor concentración de la dirección de las tesis en un reducido número de profesores:

Se constata que 6 de las tesis defendidas y 5 de las tesis en curso son dirigidas por 2 profesores [Doctorado UFRO, p. 6].

Desde la acreditación anterior mejoró la distribución de los profesores guías de tesis, no obstante, aún se evidencia una concentración en cinco académicos de claustro, quienes están guiando el $51 \%$ de las tesis en desarrollo [Doctorado PUC, p. 6].

Sobre la experiencia en dirección de tesis, solo cuatro académicos del claustro han dirigido tesis de nivel doctoral en los últimos diez años [Doctorado UPLA, p. 7].

La otra debilidad importante de los programas analizados se relaciona con el número de profesores que sustentan cada línea de investigación ofertada:

Se constata que la línea de educación y trabajo la integra un académico de trayectoria del Claustro y la reciente graduada del mismo Programa. Además, no existen académicos en categoría de colaboradores que apoyen esta línea [Doctorado UAH/UDP, p. 7].

La única línea de investigación declarada por el Programa se encuentra sustentada, al menos, por dos miembros del Claustro, sin embargo, esta situación no se replica al análisis de sustentabilidad por área temática, donde solo las áreas de Didáctica e Informática Educativa poseen un cuerpo académico de soporte con, al menos, dos profesores [Doctorado UDEC, p. 8].

De las líneas de investigación declaradas, se constata que la de Formación de profesores, no cuenta con ningún académico que la sustente. De los académicos que sustentan la línea de modelamiento, una no satisface la orientación de productividad del comité. Por último, existe una académica que adscribe a líneas de investigación distinta a las declaradas por el programa [Doctorado PUCV, p. 6].

En otros casos se advierte que no existe convergencia entre las líneas de investigación de los programas y las de los investigadores adscritos a cada doctorado:

Se evidencia que existen ocho académicos que no se encuentran adscritos a ninguna de las líneas de investigación declaradas [por el doctorado] [Doctorado UPLA, p. 7].

No se constata una apropiada coherencia entre las líneas de investigación del Programa y las áreas de especialización de los académicos. Asimismo, se observa disparidad en el nivel de desarrollo de las líneas de investigación [Doctorado UFRO, p. 6].

Así, se evidencia que algunos posgrados deben fortalecer la cantidad y calidad de los docentes, lo que requerirá contratar más doctores o articular de mejor manera la productividad de los mismos con las líneas de investigación ofertadas por cada programa, avanzando hacia una mayor profesionalización del investigador en educación en Chile (Capocasale, 2015; Gorostiaga, 2017; Palamidessi et al., 2014). 


\section{Recursos de apoyo}

Según la información que se resume en la tabla 10, la principal debilidad del criterio, se relaciona con la insuficiente cantidad de recursos destinados para becas de los doctorandos, especialmente en lo relativo a la manutención, ya que la mayoría de los programas cuentan con becas de matrícula.

Tabla 10. Recursos de apoyo doctorados en Educación con acuerdo de acreditación.

\begin{tabular}{lcccccccc}
\hline & & & & & & UCM/UCSC/ \\
& UAH/UDP & UDEC & UPLA & UFRO & PUC & PUCV & UCT/UBB \\
\hline Infraestructura y equipamiento & Sí & Sí & Sí & Sí & Sí & Sí & Sí \\
\hline Oferta becas propias manutención & Sí & No & No & Sí & Sí & No & No \\
\hline Existencia convenios institucionales & Sí & Sí & Sí & Sí & Sí & Sí & Sí \\
\hline
\end{tabular}

Fuente: Elaboración propia, basada en acuerdos de acreditación de cada doctorado.

Así, la necesidad de una mayor cantidad de becas de manutención se relaciona con la disponibilidad de recursos que cubran los costos generados por la dedicación exclusiva exigida a los estudiantes:

Las becas otorgadas son igualmente asimétricas entre las instituciones y en algunos casos no cubren la manutención [Doctorado UCM/UCSC/UCT/UBB, p. 6].

No se evidencian suficientes apoyos para la manutención de los estudiantes, lo cual no es consistente con el nivel de dedicación exclusiva exigido por el Programa, todos quienes, en la práctica, detentan [sic] compromisos laborales de media jornada [Doctorado UDEC, p. 9].

La totalidad de los estudiantes activos del doctorado disponen de becas, ya sean externas o internas, aunque la mayoría de las becas internas no cubren la manutención de los alumnos [Doctorado PUCV, p. 7].

Finalmente, en el único doctorado interuniversitario de nuestro estudio se destaca como una debilidad importante a la asimetría de los recursos de apoyo que cada universidad aporta al funcionamiento del programa:

Se observa cierta asimetría entre las instituciones en este aspecto en términos de equipamiento [...] la cantidad de académicos adscritos al programa por cada universidad es asimétrico [Doctorado UCM/UCSC/UCT/UBB, p. 6].

Así, la relación entre las becas y la acreditación del posgrado ha sido una característica del caso chileno desde sus inicios (Poblete et al., 2018; Reich, 2003), por ello, los recursos propios destinados a las becas otorgadas a los estudiantes deben asimilarse a los montos entregados por el Estado para aquellos doctorados acreditados, especialmente cuando se les exige una dedicación exclusiva al programa, lo que en ocasiones se traduce en una reducción de su jornada laboral y, por ende, de sus salarios. 


\section{Capacidad de autorregulación}

En este último criterio las debilidades se relacionan con la incapacidad de algunos doctorados para superar las debilidades detectadas en los procesos de acreditación anterior:

Aún se perciben debilidades como: los mecanismos de comunicación entre el cuerpo académico y el Comité del Programa, los altos tiempo de permanencia; la baja tasa de graduación y la alta concentración de las tesis en pocos miembros del Claustro [Doctorado PUC, p. 7].

Entre las debilidades que se mantienen desde la última acreditación, se encuentra el nivel de internacionalización y el desarrollo asimétrico de las líneas de investigación [Doctorado PUCV, p. 7].

Aún se perciben debilidades en la línea de educación y trabajo, lo que se constata, en que no existen seminarios obligatorios en esta línea y que, además, se encuentra sustentada en dos académicos, de los cuales una está recientemente graduada. Esta debilidad se mantiene, desde su primer proceso de acreditación en el año 2013 [Doctorado UAH/UDP, p. 8].

Los planes de mejora son un elemento distintivo del proceso de acreditación chileno, evidenciando un aspecto clave del aseguramiento de la calidad de los posgrados, estableciendo las prioridades que cada programa debe asumir para su mejora continua. La ineficiente gestión de dichos planes habitualmente se traduce en una profundización de las debilidades identificadas en los procesos de acreditación, transformándose en un desafío clave para la capacidad de autorregulación del sistema chileno (Zapata, 2004).

\section{Conclusiones}

El análisis de las debilidades y retos de los doctorados formadores de investigadores en educación en Chile, desarrollado en este trabajo, establece que el 80\% de dichos posgrados no están acreditados por la CNA (Corbalán et al., 2011; Ministerio de Educación, 2014); además, los cuatro programas que sí están acreditados poseen niveles de calidad intermedio-bajo, según los actuales criterios de evaluación de la CNA (Baeza, 2017), lo que hace necesario fortalecer a corto y mediano plazo algunos aspectos clave, que consoliden un mayor aseguramiento de la calidad del doctorado en Educación en Chile (Atria, 2012; Salazar, 2012; Walczak et al., 2017; Lemaitre, 2018; Poblete et al., 2018).

Uno de los ámbitos prioritarios que debe fortalecerse para garantizar la calidad de los doctorados en Educación en Chile es la mayor consistencia entre los objetivos y el perfil de egreso declarados por cada posgrado, fortaleciendo la articulación con su estructura curricular (Núñez-Valdés y González, 2019).

También debe fortalecerse la consistencia entre las líneas de investigación desarrolladas por los profesores adscritos a cada doctorado y el perfil de egreso del mismo (Gorostiaga, 2017; Miranda, 2016; Perines y Murillo; 2017). Asimismo, dichas líneas de investigación no están sustentadas por el número mínimo de investigadores 
exigido por la CNA como estándar de calidad (Palamidessi et al., 2014; Capocasale, 2015; Gorostiaga, 2017; Baeza, 2018).

Además es necesario perfeccionar los procedimientos de postulación y selección de postulantes (Corvalán et al., 2011; Miranda, 2016), asegurando una mayor calidad en la toma de decisiones al momento de aceptar un postulante, especialmente cuando no posee una formación o experiencia laboral e investigadora en el ámbito de la educación.

Asimismo, el principal reto que enfrentan los doctorados en Educación en Chile será incorporar acciones en sus planes de mejoras de corto y mediano plazo (Zapata, 2004) que garanticen mecanismos de aseguramiento de la calidad más eficientes, para avanzar hacia la obligatoriedad legal de acreditar estos programas de posgrado (Corbalán et al., 2011; Núñez-Valdés y González, 2019), al igual como ocurre en Chile con los programas de pedagogía a nivel de pregrado.

Finalmente, algunas proyecciones de la investigación pueden estar relacionadas con la utilización de un enfoque cualitativo, mediante entrevistas y focus groups, que analicen la percepción y valoración de la calidad de cada programa, desde la perspectiva de diferentes actores relevantes de los doctorados, tales como profesores, estudiantes, autoridades académicas y directores de tesis, entre otros (Perines y Murillo, 2017; Núñez-Valdés y González, 2019).

\section{REFERENCIAS}

Adrogué, C., García, A., Carranza, M. y Salto, D. (2019). Las universidades frente al aseguramiento de la calidad y las políticas de financiamiento de la investigación: estudios de caso en el sector privado argentino. Revista de la Educación Superior, 48(190), 45-70. Recuperado de: http://resu.anuies.mx/ojs/index.php/resu/article/ view/711/286.

Atria, R. (2012). Tendencias de la educación superior: el contexto del aseguramiento de la calidad. Santiago: CINDA-RIL Editores.

Baeza, P. (2017). Diversidad y diversificación en la oferta de programas de doctorado en Chile. Calidad en la Educación, (47), 179-214. http://dx.doi.org/10.4067/ S0718-45652017000200179.

Baeza, P. (2018). Programas doctorales en Chile: una propuesta tipológica para interpretar su diversidad. Calidad en la Educación, (49), 188-233. https://dx.doi. org/10.31619/caledu.n49.580.

Cabrera, D. (2013). La investigación educativa en tres revistas académicas mexicanas 1992-2002. Asignaciones de sentido. Perfiles Educativos, 35(142), 108-125. https:// doi.org/10.1016/S0185-2698(13)71852-X.

Cáceres, P. (2003). Análisis cualitativo de contenido: una alternativa metodológica alcanzable. Psicoperspectivas, 2(1), 53-82. Recuperado de: http://www. psicoperspectivas.cl/index.php/psicoperspectivas/ article/viewFile/3/3.

Capocasale, A. (2015). ¿Cuáles son las bases epistemológicas de la investigación educativa? En L. Abero, L. Berardi, A. Caposale, S. García y R. Rojas. Investigación educativa. Abriendo puertas al conocimiento (pp. 32-47). Montevideo: Consejo Latinoamericano de Ciencias Sociales (Clacso). Recuperado de: http://biblioteca.clacso.edu.ar/clacso/ se/20150610045455/InvestigacionEducativa.pdf.

Centro de Investigación Avanzada en Educación (CIAE) (s. f.). Folleto informativo. Recuperado de: http://www.ciae.uchile.cl/download. php?file=recursos $/$ brochure_ciae_2017.pdf. 
Colina, A. (2011). El crecimiento del campo de la investigación educativa en México. Un análisis a través de sus agentes. Perfiles Educativos, 33(132), 10-28. Recuperado de: http://www.scielo.org.mx/pdf/peredu/v33n132/ v33n132a2.pdf.

Comisión Nacional de Acreditación (CNA) (2017). Resolución de Acreditación de Postgrado N 904: Doctorado en Educación, Pontificia Universidad Católica de Chile. Recuperado de: https://www.cnachile.cl/ res/post/Resol.\%20N\%C2\%B0904\%20Doct.\%20 Educaci $\%$ C3\%B3n, \%20PUC.pdf.

Comisión Nacional de Acreditación (CNA) (2017). Resolución de Acreditación de Postgrado N ${ }^{\circ}$ 912: Doctorado en Educación, Universidad de Concepción. Recuperado de: http://www.saca.cnachile.cl/public/assets/postgrado/DocumentosProcesosAcreditacion/ POST-00294-02-00/NOTIFICACI \%C3\%93N\%20 DECISI \%C3\%93N//UN003-POST-00294-02RESD-1.pdf.

Comisión Nacional de Acreditación (CNA) (2013). Resolución Exenta DJ Nº 006-4 que aprueba Criterios para la Acreditación de Programas de Postgrado. Recuperado de: https://www.cnachile.cl/Documentos $\% 20$ de $\% 20$ Paginas/Criterios $\% 20$ vigentes $\% 20$ para $\% 20$ la $\% 20$ Acreditaci $\%$ C3 $\%$ B 3n $\% 20$ de $\% 20$ programas $\% 20$ de $\% 20$ postgrado $\% 20 \mathrm{a} \% 20$ partir $\% 20$ del $\% 20$ 04\%20de \%20noviembre\%20del $\% 202013$.pdf.

Comisión Nacional de Acreditación (CNA) (2015). Resolución de Acreditación de Postgrado $\mathrm{N}^{\circ}$ 722: Doctorado en Ciencias de la Educación, Universidad de la Frontera. Recuperado de: https://www.cnachile.cl/res/post/ RESOLUCI $\% C 3 \% 93 \mathrm{~N} \% 20 \mathrm{~N} \% \mathrm{C} 2 \% \mathrm{~B} 0722 \% 20$ DOC $\% 20$ EN $\% 20$ CIENCIAS $\% 20$ DE $\% 20$ LA $\% 20$ EDUCACI $\%$ C3\%93N\%20UFRO.pdf.

Comisión Nacional de Acreditación (CNA) (2016). Resolución de Acreditación de Postgrado $\mathrm{N}^{\circ}$ 487: Doctorado en Política y Gestión Educativa, Universidad de Playa Ancha de Ciencias de la Educación. Recuperado de: https://www.cnachile.cl/res/post/Resol. $\% 20$ N\%C2\%B0847\%20Doct.Pol $\%$ C3\%ADticas $\% 20$ y $\% 20$ Gesti $\%$ C3\%B3n $\% 20$ Educativa $\% 20$ UPLA.PDF.

Comisión Nacional de Acreditación (CNA) (2016). Resolución de Acreditación de Postgrado No 846: Rectifica resolución de Acreditación de Postgrado $\mathrm{N}^{\circ} 815$, relativa al proceso de acreditación del Pro- grama de Doctorado en Didáctica de la matemática, impartido por la Pontificia Universidad Católica de Valparaíso. Recuperado de: https://www.cnachile.cl/ res/post/846\%20pucv.pdf.

Comisión Nacional de Acreditación (CNA) (2016). Resolución de Acreditación de Postgrado No 861: Doctorado en Educación, Universidad Católica del Maule en conjunto con la Universidad Católica de la Santísima Concepción, Universidad Católica de Temuco y Universidad del Biobío. Recuperado de: https://www. cnachile.cl/res/post/Resol. $\% 20 \mathrm{~N} \% \mathrm{C} 2 \% \mathrm{~B} 0861 \% 20$ Doctorado $\% 20$ en $\% 20$ Educaci $\%$ C3\%B3n, $\% 20$ UCMUCSC-UCT-UBB.PDF.

Comisión Nacional de Acreditación (CNA) (2017). Resolución Exenta No 943 de Acreditación de Postgrado: Doctorado en Educación impartido por la Universidad Alberto Hurtado en conjunto con la Universidad Diego Portales. Recuperado de: http://www.saca. cnachile.cl/public/assets/postgrado/DocumentosProcesosAcreditacion/POST-00610-03-00/ NOTIFICACI $\%$ C3\% $\% 3$ N $\% 20 D E C I S I \% C 3 \% 93 \mathrm{~N} / /$ UAHURTADO-POST-00610-03-RESD-1.pdf.

Corvalán, J., Falabella, A. y Rojas, M. (2011). El doctorado en Educación: un ejemplo de desregulación en el campo en el campo de la educación superior en Chile. Calidad en la Educación, (34), 15-42. http://dx.doi. org/10.4067/S0718-45652011000100002.

Corvalán, J. y Rufinelli, A. (2007). ¿Cómo está la investigación educativa en Chile? Revista de Educación, (329), 18-26. Recuperado de: http://bibliorepo.umce.cl/ revista_educacion/2007/329/18_26.pdf.

González, H. y Jiménez, A. (2014). Inserción laboral de nuevos investigadores con grado de doctor en Chile. Journal of Technology Management \& Innovation, 9(4), 132-148. http://dx.doi.org/10.4067/S071827242014000400010.

Gorostiaga, J. (2017). La formación de investigadores en el campo de la política educativa: una mirada regional. Revista de la Educación Superior, 46(183), 37-45. https:/ / doi.org/10.1016/j.resu.2017.06.001.

Lemaitre, M. (2018). El aseguramiento interno de la calidad en América Latina. Desafíos para el siglo XXI. En Comisión Nacional de Acreditación (2018). Aseguramiento de la calidad de programas de doctorado: desafios y convergencias para Iberoamérica. Seminario Internacional. 
Serie Estudios sobre Acreditación (pp. 16-22). Recuperado de: http://www.investigacion.cnachile.cl/.

Lemaitre, M., Maturana, M., Zenteno, E. y Alvarado, A. (2012). Cambios en la gestión institucional en universidades, a partir de la implementación del sistema nacional de aseguramiento de la calidad: la experiencia chilena. Calidad en la Educación, (36), 21-52. http:// dx.doi.org/10.4067/S0718-45652012000100001.

Martí-Noguera, J., Licandro, Ó. y Gaete-Quezada, R. (2018). La responsabilidad social de la educación superior como bien común. Concepto y desafíos. Revista de la Educación Superior, 47(186), 1-22. Recuperado de: http://resu.anuies.mx/ojs/index.php/resu/article/ view/353.

Ministerio de Educación (2014). Estudio evaluativo de un conjunto de programas de doctorado chileno 2013-2014. Santiago de Chile: Mineduc. Recuperado de: http://dfi.mineduc.cl/usuarios/MECESUP/ File/2018/publicaciones/estudio-doctorados/EstudioDoctorados260418.pdf.

Ministerio de Educación (2018). Base de datos de oferta académica. Recuperado de: https://www.mifuturo.cl/ bases-de-datos-de-oferta-academica/.

Miranda, C. (2016). Acreditación de programa de postgrado mixto (académico-profesional) en Chile: impresiones académicas para un debate pendiente. Revista da FAEEBA. Educação e Contemporaneidade, 25(47), 51-62.

Muga, A. (2018). Desafíos para el aseguramiento de la calidad en programas doctorales. En Comisión Nacional de Acreditación (2018). Aseguramiento de la calidad de programas de doctorado: desafíos y convergencias para Iberoamérica. Seminario Internacional. Serie Estudios sobre Acreditación (pp. 10-15). Recuperado de: http:// www.investigacion.cnachile.cl/.

Murillo, F. J. y Perines, H. (2017). ¿Cómo los docentes no universitarios perciben la investigación educativa? Revista Complutense de Educación, 28(1), 81-99. http:// dx.doi.org/10.5209/rev_RCED.2017.v28.n1.48800.

Núñez-Valdés, K. y González, J. (2019). Perfil de egreso doctoral: una propuesta desde el análisis documental y las expectativas de los doctorandos. IE Revista de Investigación Educativa de la Rediech, 10(18), 161-175. http:/ / dx.doi.org/10.33010/ie_rie_rediech.v10i18.604.

Palamidessi, M., Gorostiaga, J. y Suasnábar, C. (2014). El desarrollo de la investigación educativa y sus vincu- laciones con el gobierno de la educación en América Latina. Perfiles Educativos, 36(143), 49-66. doi: 10.1016/ S0185-2698(14)70609-9.

Patiño, J. (2019). Análisis comparativo entre el doctorado profesional y de investigación en México. Revista Iberoamericana de Educación Superior, 10(28), 25-41. doi: https://doi.org/10.22201/ iisue.20072872e.2019.28.427.

Perines, H. y Murillo, F. J. (2017). ¿Cómo mejorar la investigación educativa? Sugerencias de los docentes. Revista de la Educación Superior, 46(181), 89-104.

Poblete, D., Baeza, P. y Droguett, N. (2018). Aseguramiento de la calidad de los programas doctorales en Chile: estado actual y desafíos. En Comisión Nacional de Acreditación (2018). Aseguramiento de la calidad de programas de doctorado: desafíos y convergencias para Iberoamérica. Seminario Internacional. Serie Estudios sobre Acreditación (pp. 67-76). Recuperado de: http:// www.investigacion.cnachile.cl/.

Rama, C. (2006). La tercera reforma de la educación superior en América Latina. Buenos Aires: Fondo de Cultura Económica (EFE).

Reich, R. (2003). Acreditación de los posgrados en Chile. Revista Calidad en la Educación, (18), 61-77. http:// dx.doi.org/10.31619/caledu.n18.386.

Salazar, J. M. (2012). Modelos de aseguramiento de la calidad en la educación superior. Santiago: CINDA-RIL Editores. Servicio de Información de Educación Superior (SIES) (2019). Informes de matrícula en la educación superior en Chile. Recuperado de: https://www.mifuturo.cl/ informes-de-matricula/.

Silva, M. (2016). Acreditación y aseguramiento de la calidad en la educación superior. Elementos analíticos para una reforma en Chile. Documento de trabajo $N^{\circ}$ 5. Chile: Centro de Investigación para la Educación Superior (CIES), Universidad San Sebastián. Recuperado de: http://www.uss.cl/wp-content/uploads/2017/06/ Serie-creacio $\%$ CC $\% 81$ n-documento-de-trabajo$\mathrm{n} \% \mathrm{C} 2 \% \mathrm{~B} 0-5$-Acreditacio $\% \mathrm{CC} \% 81 \mathrm{n}$-y-aseguramiento-de-la-calidad-en-la-educacio $\% \mathrm{CC} \% 81 \mathrm{n}$-superior. pdf.

Tornero, B., Epstein, L. y Vicuña, M. I. (2016). Consistencia entre percepciones de estudiantes sobre la calidad de sus doctorados y la evaluación de CNA. Cuadernos de investigación. Aseguramiento de la calidad en Educación 
Superior (3). Santiago de Chile: Comisión Nacional de Acreditación (CNA). Recuperado de: https://www. cnachile.cl/Biblioteca $\% 20$ Documentos $\% 20 \mathrm{de} \% 20$ Interes/Cuaderno\%203_Tornero-Digital.pdf.

Toro, J. (2012). Gestión interna de la calidad en las instituciones de educación superior. Santiago: CINDA-RIL Editores.

Walczak, M., Detmer, A., Zapata, G., Lange, M. y Reyes, M. (2017). Acreditación de doctorados vinculados a la industria: análisis de buenas prácticas internacionales y lineamientos para su desarrollo en Chile. Cuadernos de investigación. Aseguramiento de la calidad en Educación Superior (8). Santiago de Chile: Comisión Nacional de Acreditación (CNA). Recuperado de: https://www. cnachile.cl/Biblioteca $\% 20$ Documentos $\% 20 \mathrm{de} \% 20$ Interes/Cuaderno_Walczak.pdf.

Zapata, G. (2004). Acreditación institucional en Chile: una opción emergente. Calidad en la Educación, (21), 141154. http://dx.doi.org/10.31619/caledu.n21.327.

Cómo citar este artículo:

Gaete Quezada, R. (2020). Aseguramiento de la calidad de doctorados en Educación en Chile. IE Revista de Investigación Educativa de la REDIECH, 11, e740. doi: http://dx.doi.org/10.33010/ie_rie_rediech.v11i0.740. 